\title{
Toward Optimal Control of Flat Plate Photobioreactors: the Greenhouse Analogy?
}

\author{
G. van Straten*, P.M. Slegers*, L.G. van Willigenburg*, \\ R. Bosma**, A.J.B. van Boxtel*, R.H. Wijffels** \\ *Wageningen University, Systems and Control, 6708 WG Wageningen, \\ The Netherlands (Tel.+31317483331; e-mail: gerrit.vanstraten@wur.nl) \\ ** Wageningen University, Bioprocess Engineering,, 6703 HD Wageningen,
} The Netherlands

\begin{abstract}
The cultivation of algae in photo-bioreactors shows similarities to crop cultivation in greenhouses, especially when the reactors are driven by sun light. Advanced methodologies for dynamic optimization and optimal control for greenhouses are known from earlier research. The aim here is to extend these methodologies to microalgae cultivated in a flat plate photo-bioreactor. A one-state space model for the algal biomass in the reactor is presented. The growth rate vs. light curve is parameterized on the basis of experimental evidence. Spatial distribution of light and growth rate between the plates is also considered. The control variable is the dilution rate. Dynamic optimal control trajectories are presented for various choices of goal function and external solar irradiation trajectories over a horizon of 3 days. It was found that the algae present in the reactor at final time represent a value for the future. Numerical and theoretical results suggest that the control is bang-(singular-)bang, with a strong dependence on the weather. The optimal biomass also depends on the available light, and achieving it to reach a new optimal steady cycle after a prolonged change in weather may take several days. A preliminary theoretical analysis suggests a control law that maximizes the effective growth rate. The analysis shows that like in the greenhouse case, the co-state of the algal biomass plays a pivot role in developing on-line controllers.
\end{abstract}

Keywords: Optimal control; Photo-bioreactor; Microalgae; Dynamic Optimization; State-space model; Bang-bang control.

\section{INTRODUCTION}

Algal biomass production is receiving considerable attention because of the potential for the production of valuable chemicals for food supplements as well as lipids that can be a source of sustainable bio-fuels. Most studies on algae focus on the physiology of biomass and valuable substance production, and on the design of suitable bio-reactors (Barbosa et al. 2005; Bosma 2010; Cuaresma et al. 2009; Janssen et al. 2003; Richmond and Cheng-Wu 2001). The control aspects of photo-bioreactors (PBRs) have received much less attention, and focussed on $\mathrm{pH}$ control (Berenguel et al. 2004) or $\mathrm{CO}_{2}$ supply (Buehner et al. 2009) .

Over the past years, considerable efforts have been devoted to the development of an optimal control strategy for crop production in greenhouses (van Straten et al., in press, 2010) This consists essentially out of two hierarchical steps: (i) dynamic optimization, to be performed with smooth, assumed nominal weather, and (ii) on-line receding horizon optimal control. The dynamic optimization delivers a co-state trajectory of the biomass, which represents the marginal value of an extra unit of biomass at any time. It can subsequently be used to derive suitable on-line control laws. The approach requires (i) a dynamical state space model of the system, (ii) the formulation of an suitable (economic) goal function, (iii) the forecast of the external variables (weather), (iv) a suitable solution method.

This paper explores the applicability of this methodology for control of a flat plate PBR. We first derive a model, formulate a goal function, and then solve the control problem for two sample light patterns over 3 days. Numerical results as well as a preliminary analysis are presented that shed light on the optimal control problem of the PBR. Finally, similarities and difference with the greenhouse case are briefly discussed, suggesting directions for further investigations.

\section{THE PBR BIOMASS MODEL}

This paper focuses on the biomass production. At this stage, the generation of the final chemical valuables, which constitutes a more elaborate control problem, is not yet explored. It is assumed that supply of $\mathrm{CO}_{2}$ and nutrients are non-limiting, and that surplus dissolved oxygen is removed with a rate that is sufficient to prevent growth inhibition. Temperature and $\mathrm{pH}$ are assumed to be ideally controlled at a level considered optimal for the algal type studied. The reactor consists of vertically placed transparent sheets in 
which the biomass broth is contained, ideally mixed by air flow agitation. The reactor receives time varying diffusive and direct sunlight on both sides. A light model is used to derive the photon flux towards the algae as a function of measured direct and diffuse sunlight. Light irradiance inside the reactor is attenuating towards the centre according to Lambert-Beer

$I(z, t)=I_{o}(t) e^{-\varepsilon(t) z}+I_{d}(t) e^{-\varepsilon(t)(d-z)}$

where $I_{o}(t)$ and $I_{d}(t)$ are the incident radiation through the vertical flat plates in $\mu \mathrm{mol}[\mathrm{phot}] \mathrm{s}^{-1} \mathrm{~m}^{-2}$ at time $t$ at $z=0$ and $z=d$, respectively, with $d$ the distance (in $\mathrm{m}$ ) between the plates, and $\varepsilon(\mathrm{t})$ the extinction coefficient $\left(\mathrm{m}^{-1}\right)$, given by the self-shading equation

$\varepsilon(t)=\varepsilon_{o}+\alpha C_{X}(t)$

in which $\varepsilon_{o}$ is the extinction coefficient of the cultivation medium $\left(\mathrm{m}^{-1}\right), C_{X}$ is the algal biomass $\left(\mathrm{g}[\mathrm{dw}] \mathrm{m}^{-3}\right)$, and $\alpha$ the absorption coefficient $\left(\mathrm{m}^{2} \mathrm{~g}^{-1}[\mathrm{dw}]\right)$. The dependency of the growth rate is modeled according to the Steele equation

$f(I(z, t))=\frac{I(z, t)}{I_{s}} \exp \left(1-\frac{I(z, t)}{I_{s}}\right)$

where $I_{s}$ is the irradiance at which the growth rate is maximal. Define the effective growth rate $\left(\mathrm{d}^{-1}\right)$ at temperature $T$ as

$$
\begin{aligned}
& \mu_{T}\left(I_{o}(t), I_{d}(t), C_{X}(t)\right)= \\
& \mu_{\max } \frac{1}{h d} \int_{z=0}^{d} \int_{x=0}^{h} f_{p}\left\{I\left[x, z, t, C_{X}(t)\right], T_{s p}\right\} d x d z
\end{aligned}
$$

where $x$ is the vertical dimension to height $h$ and $z$ the horizontal dimension to thickness $d$. Defining the maintenance rate $\left(\mathrm{d}^{-1}\right)$ at temperature $T$ as $k_{m T}$, then the algal biomass dynamics is given by

$$
\frac{d C_{X}(t)}{d t}=\left(\mu_{T}\left(I_{o}(t), I_{d}(t), C_{X}(t)\right)-k_{m T}-D(t)\right) C_{X}(t)
$$

where $D(t)$ is the dilution rate $\left(\mathrm{m}^{3}[\right.$ water $] \mathrm{m}^{3}[\mathrm{rv}] \mathrm{d}^{-1} ;$ rv stands for reactor volume). Note that (4) integrates the growth rate over the horizontal optical path and over the height of the reactor. In this paper, for simplicity, the vertical light distribution (relevant for direct light and shading) is encapsulated in the calculation of a representative uniform irradiance at the surface. The simple one-state model (5) does not describe any adaptation of the parameters to prolonged light regimes.

The model is parameterized on data from Bosma et al., (2007) for the alga Monodus subterraneus. In particular Fig. 1 shows the behaviour of the Steele equation, viz. some data at the optimal temperature $23.5^{\circ} \mathrm{C}$, and the polynomial curve presented by Bosma. The parameter values used in all calculations are $d=0.02 \mathrm{~m}, \mu_{\max T}=0.9 \mathrm{~d}^{-1}, k_{m T}=0.1 \mathrm{~d}^{-1}$, $I_{s}=350 \mu \mathrm{mol}[\mathrm{phot}] \mathrm{s}^{-1} \mathrm{~m}^{-2}, \varepsilon_{o}=2 \mathrm{~m}^{-1}, \alpha=0.15 \mathrm{~m}^{2} \mathrm{~g}^{-1}[\mathrm{dw}]$. It is nice to note that the saturation light value coincides with values derived from production experiments for 'warm' algae in Lake Balaton (van Straten and Herodek, 1982).

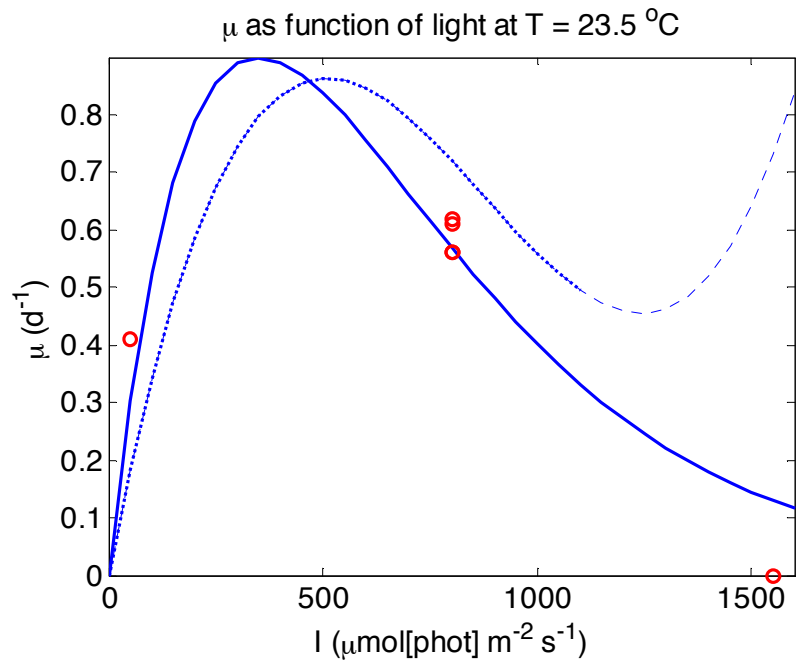

Fig. 1. Parameterization of the Steele function (solid line), compared to data ('o') and polynomial approximation.

\section{THE OPTIMAL CONTROL PROBLEM}

By defining the state $x=C_{X}$, control input $u=D$, external inputs $v=\left[I_{o}, I_{d}\right]^{T}$, and the parameters $p$ as above, (5) can be written in standard state space form

$\dot{x}=f(x, u, v, p)$

with $f$ given by obvious substitutions in the right hand side of (5). A possible goal for the operation of the PBR is to maximize volumetric productivity, which leads to the goal function

$J=\int_{t_{o}}^{t_{f}} D C_{X} d t$

or

$J=\int_{t_{o}}^{t_{f}} L(x, u) d t$ with $L=u x$

The optimal control problem is to find the optimal control trajectory $u^{*}(t)$ that maximizes $J$ subject to the control constraint,

$0 \leq u(t) \leq 2$

determined by the maximum dilution rate (set at $2 \mathrm{~d}^{-1}$ here). The computation of the trajectory requires the specification of a nominal light trajectory. Case $\mathrm{A}$ is a repetitive three day pattern, shown in the graphs below, and case B assumes that the irradiance on the second day is only half of standard.

\section{RESULTS}

Numerical calculations were done in Matlab with the tomlab/propt toolbox (Tomlab Optimization $\mathrm{AB}$, Västerås, Sweden). All trajectories are approximated by polynomials 
with a collocation method, which turns the problem into a standard NLP optimization.

\subsection{No final constraint}

Figure 2 shows the external inputs (sum of light on both sides), the optimal control trajectory, and the associated biomass pattern. The goal function value $J$ in $\mathrm{gm}^{-3}[\mathrm{rv}]$ over 3 days is presented in the figure title bar. It is clear that without restrictions on the minimum algae biomass, it is optimal to harvest all algae still present in the reactor near the final time. The time the draining starts depends upon the control constraint (not shown). Clearly, in a continuous cultivation this is not what we want, as in this way there is no biomass left to produce in the days to come (of which the optimization has no knowledge). In order to avoid this behaviour it is conceivable to attach a value to the remaining algal concentration at final time, which can be seen as an investment to the future. This reminds to the situation in greenhouses, where leaves represent a value to ensure future production.

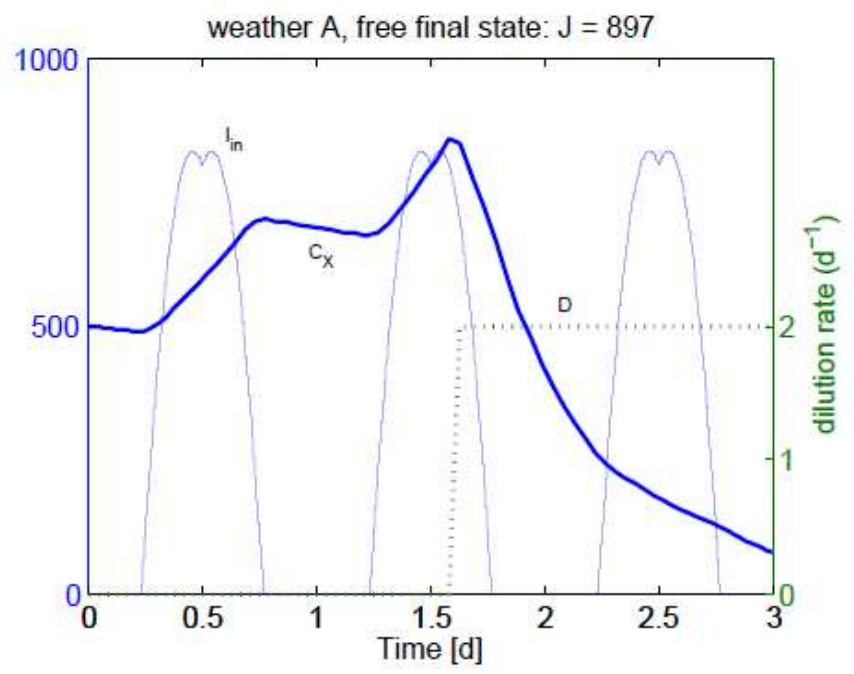

Fig. 2. Optimal control with free final state. Left scale: Total incident irradiation $\left(I_{n} \mu \mathrm{mol}[\mathrm{phot}] \mathrm{s}^{-1} \mathrm{~m}^{2}\right)$, biomass $\left(C_{X} \mathrm{gm}^{-3}\right)$, right scale: dilution rate (control signal, $D \mathrm{~d}^{-1}$ ).

\subsection{Steady cycle}

One option is to assign a soft penalty to deviations of the final state of a fixed target value. The disadvantage is that the proper choice of the fixed value is not known. In order to remedy the situation, here the choice was made to enhance the problem with an additional final constraint

$x\left(t_{f}\right)=x\left(t_{o}\right)$

This creates a steady cycle of biomass. First, Table 1 shows how the optimal goal function depends upon the initial (and hence, final) condition itself. The optimal volumetric biomass yield (goal function) and the overall photon yield ( $\mathrm{g}$ $\mathrm{mol}^{-1}$ [phot]) are given as function of the biomass. The photon yield was computed by dividing the harvested biomass by the light sum absorbed between the plates. The generation of this table required several optimizations with every time a new initial/final state. It can be seen that it is not optimal to maximize the biomass. In fact, the biomass needs to be balanced for the prevailing light in such a way that the effective growth rate $\mu_{T}$, taken over the entire day, is as high as possible. The range over which the biomass can vary without much difference is quite large. However, it was observed that the optimal dilution rate pattern changes from harvesting only at the first day when the initial and final biomass are set at a high value, and harvesting only at the last day at a low value (not shown). Harvest is performed every day only when the biomass is approximately at its optimum (see also Fig. 3 below).

Next, the optimization was allowed to optimize the initial biomass itself, leading to the result shown in the table with an asterix, and with the control pattern in Fig. 3. It can be seen that the optimal solution is bang-bang (see section 5). Pumping starts at the end of the day, before sunset, and stops when it gets dark, or slightly after.

Table 1. Volumetric production and quantum yield for a steady 3 day cycle, with fixed initial and final biomass

\begin{tabular}{lll}
$\begin{array}{l}\text { Biomass } \\
C_{X}\left(\mathrm{gm}^{-3}\right)\end{array}$ & $\begin{array}{l}\text { Production } \\
J\left(\mathrm{gm}^{-3}[\mathrm{rv}]\right)\end{array}$ & $\begin{array}{l}\text { Photon Yield } \\
\left(\mathrm{g} \mathrm{mol}^{-1}[\mathrm{phot}]\right)\end{array}$ \\
\hline 500 & 685 & 0.189 \\
1000 & 846 & 0.214 \\
1250 & 856 & 0.215 \\
$1261^{*}$ & $856^{*}$ & $0.215^{*}$ \\
1500 & 851 & 0.213 \\
2000 & 811 & 0.202 \\
2500 & 706 & 0.174 \\
\hline
\end{tabular}

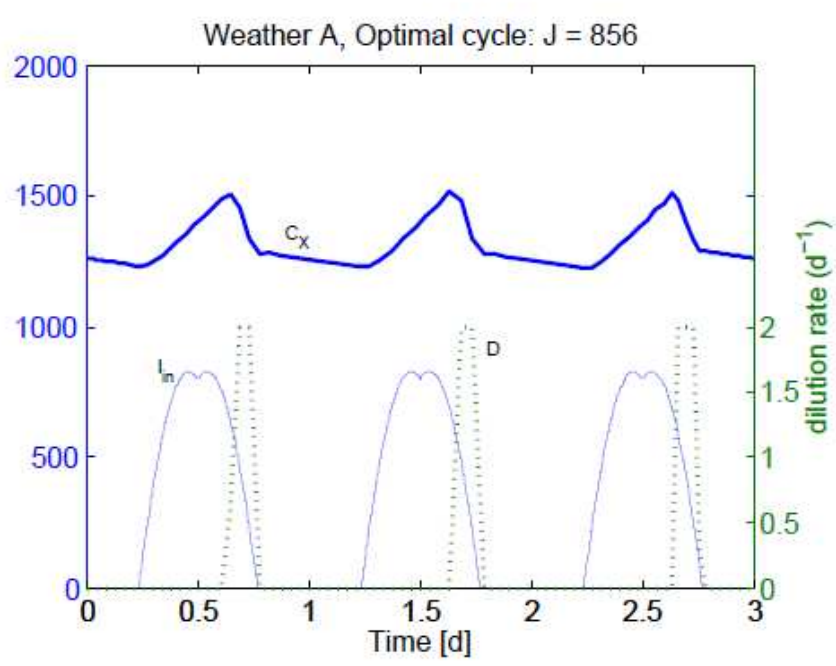

Fig. 3. Optimal control with steady cycle. Left scale: Total incident irradiation $\left(I_{n} \mu \mathrm{mol}[\mathrm{phot}] \mathrm{s}^{-1} \mathrm{~m}^{2}\right)$, biomass $\left(C_{X} \mathrm{gm}^{-3}\right)$, right scale: dilution rate (control signal, $D \mathrm{~d}^{-1}$ ). 
Next, the optimal pumping trajectory was applied to a situation where the initial concentration was not optimal. By repeating the calculation starting with the final value from the previous 3 days, a steady cycle was iteratively found. Depending upon the discrepancy between initial biomass and optimal biomass, this can take 10-20 days or more. This suggests that in an on-line situation, after a spell of dull days, thoughtless application of a canned pumping regime might result in production loss for quite some time. System (5) has time varying time constants. At zero pumping the time constant is in the order of $1 \mathrm{~d}$. On the other hand, lowering the biomass can be done with time constants that can be chosen almost at will, as the pumping rate is, theoretically, free to choose. This means that in the reverse situation, where bright days are followed by dull days, the biomass can be lowered quickly. This heuristic argument suggests that it is imperative to adjust the pumping regime to the light conditions.

\subsection{Variable weather}

The optimal pumping regime under variable weather is shown in Fig. 4. The optimization adapts the dilution rate to the prevailing conditions, and no pumping occurs on the second day. The optimal volumetric production is with 716 $\mathrm{gm}^{3}[\mathrm{rv}]$ over 3 days obviously lower than with the weather of case $\mathrm{A}$, but the quantum yield appeared to be slightly better (0.22 $\mathrm{g} \mathrm{mol}^{-1}$ [phot] $)$.

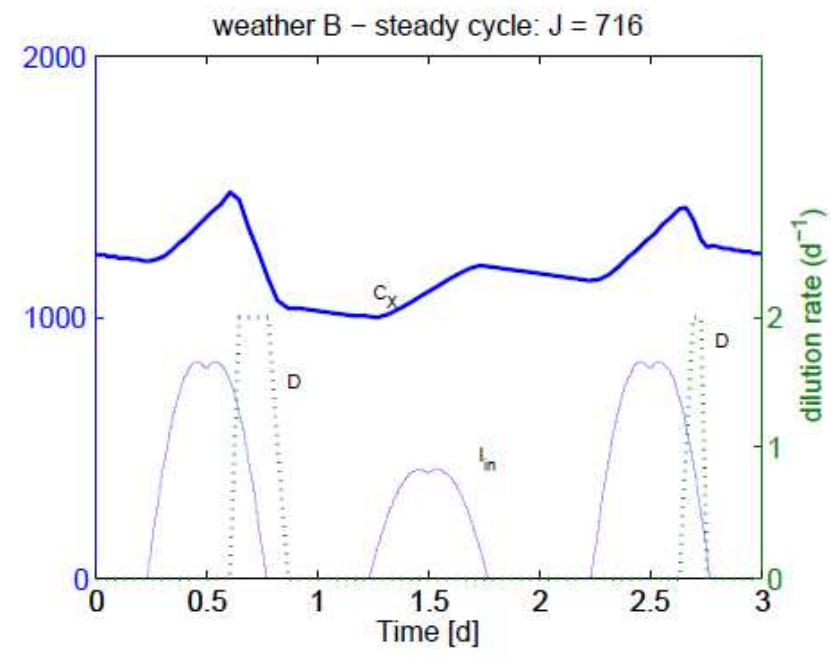

Fig. 4. Optimal control with steady cycle in response to dull day 2. Left scale: Total incident irradiation $\left(I_{n} \mu \mathrm{mol}[\mathrm{phot}] \mathrm{s}^{-}\right.$ $\left.{ }^{1} \mathrm{~m}^{2}\right)$, biomass $\left(C_{X} \mathrm{gm}^{-3}\right)$, right scale: dilution rate (control signal, $\left.D \mathrm{~d}^{-1}\right)$.

\section{THEORETICAL CONSIDERATIONS}

The dynamic optimizations performed in the previous section give insight into the problem, but require full knowledge over the complete horizon, which is not really available on-line. One possibility is to use on-line a receding horizon controller, using the same goal function, and making model predictions based on weather forecasts. It is, however, necessary to consider the 'end effect' observed above. Further work is needed to see how a value can be attached to algae left in the reactor at final time. The greenhouse experience suggests that the co-state that appears in the theory of dynamic optimal control can play a major role.

To shed some light on this, here a preliminary analysis is made. With the model equations (5), (6), and the goal function equations (7), (8), and assuming no final constraint, it can be shown that one of the necessary conditions for optimality amounts to

$-\dot{\lambda}=F_{X} \lambda+u, \quad \lambda\left(t_{f}\right)=0$

where $\lambda$ is the co-state associated to the biomass, and where

$$
F_{X}=\frac{\partial f}{\partial x}=\left(\mu_{T}\left(I_{o}, I_{d}, C_{X}\right)-k_{m T}-D\right) \frac{d \mu_{T}}{d C_{X}}
$$

This term can be determined at any time from the prevailing biomass, and the measured external disturbances. The other condition follows from setting the derivative of the Hamiltonian to $u$ equal to zero, and appears to be

$0=-x \lambda+x$

The latter condition is not a function of $u$ which implies that the solution is bang-singular-bang. However, (13) is intriguing since it suggests that $\lambda=1$ for all times except final time. This makes sense as it means that the marginal value of a unit of biomass is constant over time (and independent on the weather). However, at final time, because of (11), the costate must be zero, and this can only be not in conflict with (13) if $x\left(t_{f}\right)=0$. Hence, in the unconstraint situation, at final time the remaining algae will be harvested at infinite rate, to make the biomass zero. All this applies only when there are no constraints on the control. If no constraints are hit (11) leads to an interesting control law that takes the form

$F_{X}=-u$

and that can be worked out to

$$
D=\frac{1}{1+\frac{d \mu_{T}}{d C_{X}}}\left(\mu_{T}\left(I_{o}, I_{d}, C_{X}\right)-k_{m T}\right)
$$

This is a control law that could be applied on line, as with help of the model, and measured values of light intensity and optical density as measure of biomass, all values can be computed. In this way, (15) is a kind of self-optimizing controller. It has an appealing interpretation. When the biomass density causes the spatial mean growth rate to be to the left of the maximum of the growth rate - biomass curve, i.e. where $d \mu_{T} / d C_{X}>0$, the optimal dilution rate is lower than the net growth rate, leading to biomass increase, whereas to the right the dilution rate will be higher, bringing the biomass down. Hence, this control law would automatically adjust the biomass to a more appropriate value, which will vary dynamically, depending on the light. 
Further investigations are needed to expand the theory to the situation with a lower bound on $D$. With $u(t) \geq 0$, the necessary conditions must be expanded with an additional term caused by the constraint. It also involves and additional co-state (Stengel, 1994). These expanded conditions only apply when the constraint is on the bound, while (11) still applies when the control is off the lower bound. Transversality conditions are needed to link the off bound and on bound situations, and also the switching instants need to be found. This is the subject of further studies.

\section{COMPARISON WITH THE GREENHOUSE}

The common factor between the greenhouse cultivation control and solar driven PBR control is the wish to exploit the opportunities of the external signals as much as possible. The optimal control methodology is a powerful framework to assist in finding the best solutions. Quick and automatic adaptation to changes in economic parameters and goals is one of the major advantages of the methodology. In both the greenhouse as well as the PBR quite a number of on-line measurements are available. Also, in both cases, there is no easy direct measurement of the photosynthetic rate, which makes it necessary to use other data plus the model to optimize the plant's operation.

There are also differences. Whereas in the greenhouse timescale decomposition is possible, this is not relevant in the algal plant; conversely, the PBR shows widely time varying time constants. The situation will be more involved when also the production of valuable chemicals by stressing the algae is taken into account. And, in addition, the other control aspects like temperature control, $\mathrm{pH}$ control, $\mathrm{CO}_{2}$ supply and oxygen removal will also have to be taken into account.

Part of the power of the optimal control methodology is the consideration of co-states, that measure the sensitivity of the optimal solution to state variations (Stengel, 1994). This information is useful for the design of optimal on-line controllers.

\section{CONCLUSIONS}

The dynamic optimal control of the PBR appears to be bangsingular-bang. No switching rules have been derived yet that could guide on-line feed-back decisions on timing of pumping, but a receding horizon control should be no problem. The preliminary co-state analysis suggests that maximizing effective growth rate (thus realising maximum photon yield) in an on-line fashion might also be a good policy. In order to cope with inevitable variations in dynamic behaviour of the algae, this will require a model based observer to construct the actual growth rate from available measurements, such as acid needed for $\mathrm{pH}$ control, the oxygen production rate, and the optical density. Maximizing biomass concentration is not the best policy to maximize volumetric yield. It need to be said, however, that for reasons of downstream processing maximizing the biomass concentration can bring cost savings. Also pumping costs and nutrient costs may be relevant in some cases. The power of optimal control is that it is easy to adapt the goal function to include these and similar factors that influence the overall economic performance. It then leads immediately and automatically to a new optimal control.

\section{REFERENCES}

Barbosa, M. J., Zijffers, J. W., Nisworo, A., Vaes, W., Van Schoonhoven, J. \& Wijffels, R. H. (2005). Optimization of biomass, vitamins, and carotenoid yield on light energy in a flat-panel reactor using the A-stat technique. Biotechnology and Bioengineering, 89, 233-242.

Berenguel, M., Rodríguez, F., Acién, F. G. \& García, J. L. (2004). Model predictive control of $\mathrm{pH}$ in tubular photobioreactors. Journal of Process Control, 14, 377387.

Bosma, R. (2010) Towards high productivities of microalgae in photobioreactors. PhD thesis. Wageningen University.

Bosma, R., Van Zessen, E., Reith, J. H., Tramper, J. \& Wijffels, R. H. (2007). Prediction of volumetric productivity of an outdoor photobioreactor. Biotechnology and Bioengineering, 97, 1108-1120.

Buehner, M. R., Young, P. M., Willson, B., Rausen, D., Schoonover, R., Babbitt, G. \& Bunch, S. (2009). Microalgae growth modeling and control for a vertical flat panel photobioreactor. Proceedings of the American Control Conference, 2301-2306.

Cuaresma, M., Janssen, M., Vílchez, C. \& Wijffels, R. H. (2009). Productivity of Chlorella sorokiniana in a short light-path (SLP) panel photobioreactor under high irradiance. Biotechnology and Bioengineering, 104, 352359.

Janssen, M., Tramper, J., Mur, L. R. \& Wijffels, R. H. (2003). Enclosed outdoor photobioreactors: Light regime, photosynthetic efficiency, scale-up, and future prospects. Biotechnology and Bioengineering, 81, 193210.

Richmond, A. \& Cheng-Wu, Z. (2001). Optimization of a flat plate glass reactor for mass production of Nannochloropsis sp. outdoors. Journal of Biotechnology, 85, 259-269.

Stengel, R. F. (1994). Optimal Control and Estimation, Dover Publ, Mineola, NY, USA.

Van Straten, G. \& Herodek, S. (1982). Estimation of algal growth parameters from vertical primary production profiles. Ecological Modelling, 15, 287-311.

Van Straten, G., Van Willigenburg, L. G., Van Henten, E. J. \& Van Ootghem, R. J. C. (2010). Optimal Control of Greenhouse Cultivation, Taylor\&Francis, CRC Press. 